\title{
Recessed Oxynitride Dots on Self-Assembled Ge Quantum Dots Grown by LPD
}

\author{
P.-S. Kuo, ${ }^{a}$ B.-C. Hsu, ${ }^{a}$ P.-W. Chen, ${ }^{a}$ P. S. Chen, ${ }^{b}$ and C. W. Liu ${ }^{\text {a,b,z }}$ \\ ${ }^{a}$ Department of Electrical Engineering and Graduate Institute of Electronics Engineering, National Taiwan \\ University, Taipei, Taiwan \\ ${ }^{b}$ Electronics Research and Service Organization of the Industrial Technology Institute, Hsinchu, Taiwan
}

Recessed oxynitride dots deposited on self-assembled Ge dots are demonstrated using liquid-phase deposition (LPD). By adding ammonia into the solution, the nitrogen atoms can be incorporated into the deposited film. The tensile strain of the Si cap layer directly deposited on Ge dots can enhance the oxynitride nucleation and deposition on $\mathrm{Si}$ surface. The tensile strain may also increase the etching rate of the Si cap layer and the recessed dots are formed directly above the Ge dots. The LPD-SiON dots have a higher dot step height as compared to LPD-SiO 2 dots.

(C) 2004 The Electrochemical Society. [DOI: 10.1149/1.1785911] All rights reserved.

Manuscript submitted October 9, 2003; revised manuscript received February 28, 2004. Available electronically August 30, 2004

$\mathrm{Si} / \mathrm{SiGe}$ electro-optical devices are of great interest in optoelectronics and high-speed electronics due to their ability for integration with Si technology. The metal-oxide-semiconductor (MOS) tunneling photodetectors with $\mathrm{Ge} / \mathrm{Si}$ quantum dot absorption layers are demonstrated with low dark current and high efficiency. ${ }^{1,2}$ To avoid $\mathrm{Ge} / \mathrm{Si}$ material degradation such as strain relaxation and Ge outdiffusion during high-temperature processing, a low-temperature liquid-phase deposition (LPD) process has been used to form gate dielectrics. ${ }^{3}$ The strain-induced oxide dots are formed due to the tensile strain on the Si cap layer above the Ge quantum dots. ${ }^{4}$ In this article, oxynitride films are deposited on self-assembled Ge/Si quantum dots and the characteristics of the oxynitride dots are investigated with atomic force microscopy (AFM), secondary-ion mass spectroscopy (SIMS), and transmission electron microscopy (TEM).

$\mathrm{Ge} / \mathrm{Si}$ quantum dots were prepared by ultrahigh-vacuum chemical vapor deposition (UHVCVD) on p-type $\mathrm{Si}(001)$ substrate. After a Si buffer layer of $50 \mathrm{~nm}, 20$ periods of $\mathrm{Ge} / \mathrm{Si}$ bilayers were grown to form the self-assembled $\mathrm{Ge}$ dots at $600^{\circ} \mathrm{C}$ under StranskiKrastanov (SK) growth mode. ${ }^{5}$ The Si spacer layer between Ge dots was $20 \mathrm{~nm}$ thick. A $3 \mathrm{~nm}$ (nominal thickness) Si cap was deposited as a top layer on Ge dots for the subsequent LPD process. All layers grown by UHVCVD were unintentionally doped with an estimated hole concentration of $\sim 1 \times 10^{16} \mathrm{~cm}^{-3}$.

LPD was used for $\mathrm{SiO}_{2}$ formation with the advantages of low thermal budget, low cost, and high throughput. A simplified mechanism of LPD growth was proposed originally by Nagayama et al. ${ }^{6}$ based on the reaction of $\mathrm{H}_{2} \mathrm{SiF}_{6}$ with water to form hydrofluoric acid and solid $\mathrm{SiO}_{2}$

$$
\mathrm{H}_{2} \mathrm{SiF}_{6}+2 \mathrm{H}_{2} \mathrm{O} \leftrightarrows \mathrm{SiO}_{2}+6 \mathrm{HF}
$$

In this article, the LPD method with $\mathrm{H}_{2} \mathrm{O}$ addition only is used and the detailed experimental flow diagram of this LPD process can be found in Ref. 3 and 7. The LPD oxynitride film was deposited by adding $\mathrm{NH}_{4} \mathrm{OH}$ into the solution saturated with silica, which is different from the method proposed in Ref. 8, where additional $\mathrm{H}_{3} \mathrm{BO}_{3}$ chemicals were added.

Figure 1 shows the cross-sectional TEM image of the 20-period Ge dot structure. The base width and the height of each Ge dot are about $100 \mathrm{~nm}$ and $6 \mathrm{~nm}$, respectively. Because the spacer is smaller than the correlation length, the Ge dots are self-assembled in a row vertically in the same position. ${ }^{9}$ Above the Ge dots, the recessed LPD-SiON dots are formed by adding $0.5 \mathrm{M} \mathrm{NH}_{4} \mathrm{OH}$ during the LPD process. The typical SiON dot has a height of $22 \mathrm{~nm}$ and a wetting layer of $10 \mathrm{~nm}$ after $20 \mathrm{~min}$ growth. Note the definition of dot height is the vertical distance between the top of the dot and the wetting layer. Due to the relaxation of $\mathrm{Ge}$ dots, the $\mathrm{Si}$ cap area

z E-mail: chee@cc.ee.ntu.edu.tw directly on the Ge dots has tensile strain, while the Si cap area above the $\mathrm{Ge}$ wetting layer is strain free. During the oxynitride deposition process, the ionized $\mathrm{NH}_{4}^{+}, \mathrm{H}^{+}, \mathrm{F}^{-}$ions incorporate into the deposited film. The ions may diffuse to the Si cap/oxynitride interface and attack the Si cap surface. Therefore, the Si cap is also etched during the deposition. The tensile strain probably enhances the etching rate of Si cap directly above the Ge dots. Therefore, the oxynitride is deposited in the recess and the recessed oxynitride dot is formed (Fig. 1).

To confirm that the tensile strain can enhance the oxynitride deposition rate, both the tensile-strained $\mathrm{Si}$ sample and the control $\mathrm{Si}$ sample were studied. Figure 2 shows the oxynitride film thickness vs. deposition time for both samples and the structure of the strained $\mathrm{Si}$ sample is shown in the inset. The strained $\mathrm{Si}$ sample yielded a thicker film as compared to the control Si sample. Therefore, the enhanced deposition rate above the Ge dot probably comes from the tensile strain of the Si cap layer.

The SIMS profiles of $\mathrm{O}, \mathrm{N}$, and Ge atoms of the LPD oxynitride film with $1 \mathrm{M} \mathrm{NH}_{4} \mathrm{OH}$ in the solution are shown in Fig. 3. The SIMS profile was performed by $\mathrm{Cs}$ ion source with $2 \mathrm{keV}$ energy. The increase of Ge signal as the number of deposition layers increased indicates the size increase of the Ge quantum dots, as confirmed by TEM micrograph (Fig. 1). However, the Ge signal of the top layer is smaller as compared to the second and third Ge layer due to the top Ge layer being etched by $\mathrm{NH}_{4} \mathrm{OH}$. The estimated O:N

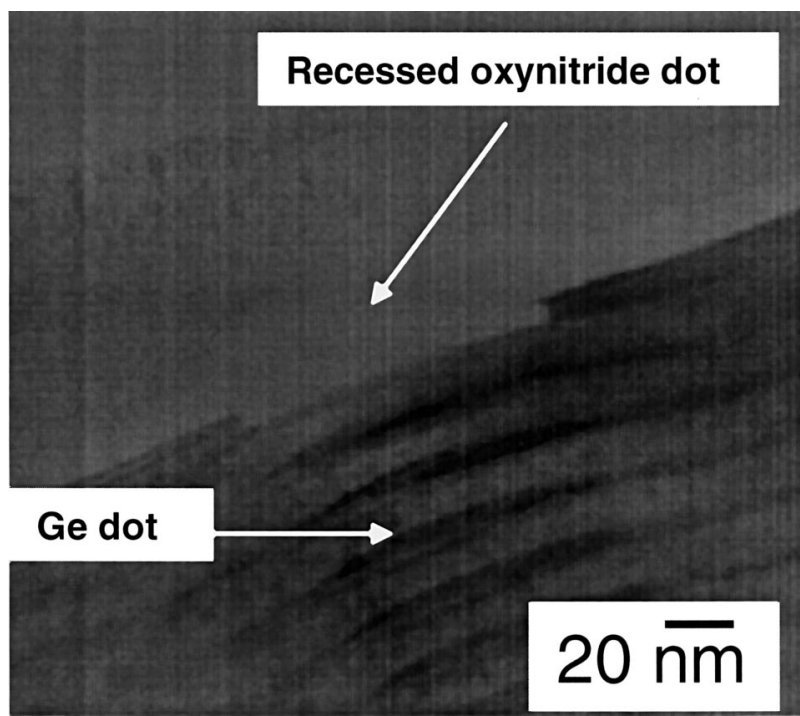

Figure 1. Cross-sectional TEM micrograph of recessed oxynitride dots on self-assembled Ge quantum dots. 


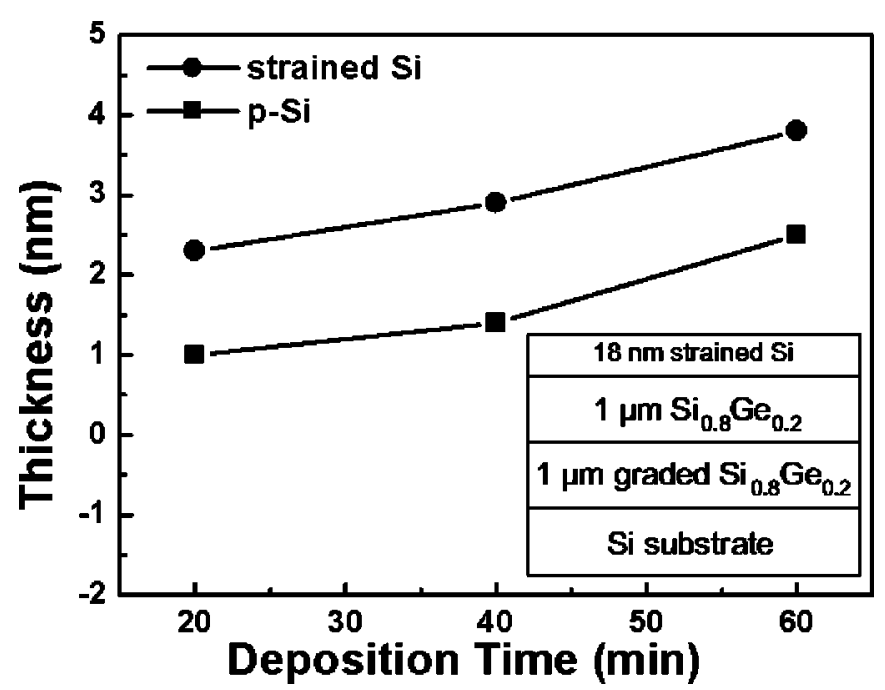

Figure 2. Oxynitride film thickness $v s$. deposition time for both tensilestrained $\mathrm{Si}$ and control Si samples. The inset shows the structure of the strained Si sample.

ratio is $16: 7$ at $\mathrm{N}$ peak position $(\sim 55 \mathrm{~nm}$ from the surface). Note that the $\mathrm{N}$ and $\mathrm{O}$ are piled up at $\sim 55 \mathrm{~nm}$, and the first Ge peak is also located at $\sim 55 \mathrm{~nm}$, indicating that both the Si cap and the top Ge layer were etched during deposition and the oxynitride/Ge interface is about $55 \mathrm{~nm}$ in depth.

From the TEM image, the oxynitride wetting layer is $10 \mathrm{~nm}$ thick, similar to the LPD-SiON thickness on the control Si samples under the same growth condition. The thickness of LPD-SiON on control Si sample can be measured by ellipsometry. The step height $(22 \mathrm{~nm})$ of the LPD-SiON dots also agrees well with the AFM results. Therefore, the AFM morphology and the film thickness measured by ellipsometry on control Si samples were used to characterize the SiON dots height and the wetting layer thickness, respectively. Figure 4a shows the average dot height of $\mathrm{LPD}-\mathrm{SiO}_{2}$ and LPD-SiON $\left(0.5 \mathrm{M}\right.$ and $\left.1 \mathrm{M} \mathrm{NH}_{4} \mathrm{OH}\right)$ dots $v s$. deposition time. The LPD-SiON had a higher deposition rate as compared to the LPD- $\mathrm{SiO}_{2}$, and the deposition rate increased as ammonia concentration increased. The dot height $v s$. wetting layer thickness is shown in Fig. 4b. Under the same wetting layer thickness, the LPD-SiON dots still yielded a higher dot height. The LPD-SiON dots with $1 \mathrm{M}$ $\mathrm{NH}_{4} \mathrm{OH}$ concentration were the tallest, and the dot height almost did

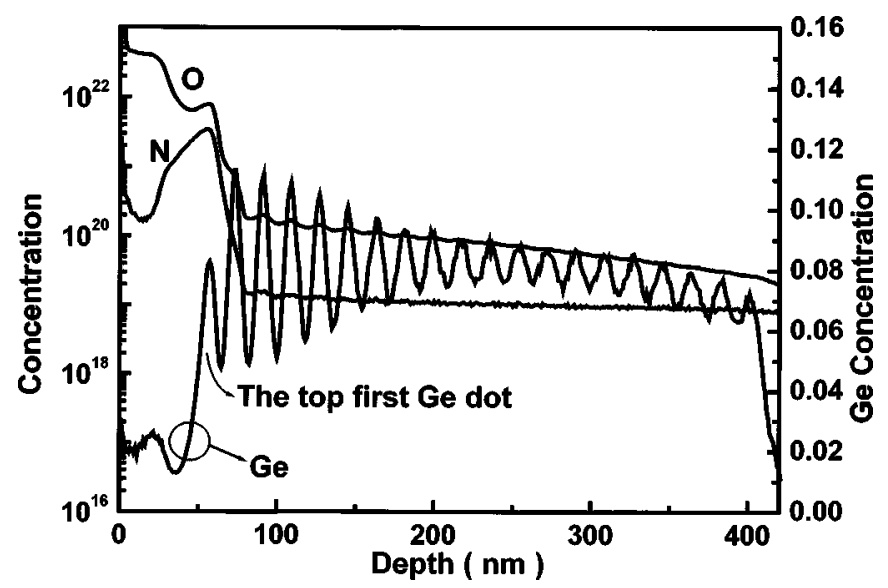

Figure 3. SIMS profiles of $\mathrm{O}, \mathrm{N}$, and Ge atoms of LPD oxynitride on 20 layer Ge quantum dots. The increase of the Ge signal as the number of deposition layers increases indicates the size increase of the Ge quantum dots, as confirmed by TEM micrograph.
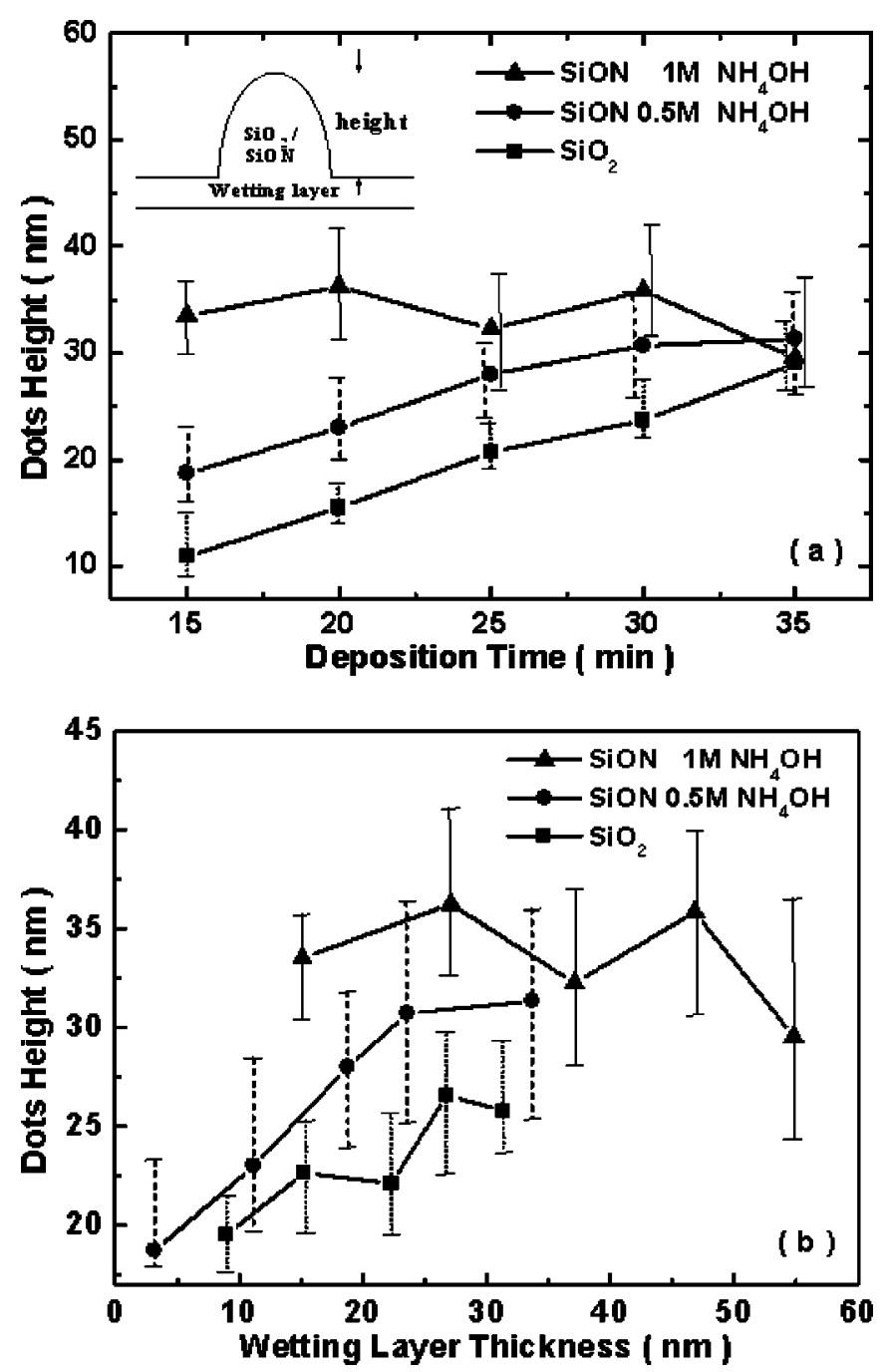

Figure 4. (a) Dot height of LPD oxide and oxynitride vs. deposition time. The measured thickness on the control Si wafers is similar to the wetting layer thickness. (b) Dot height vs. wetting layer thickness. The oxynitride dots are taller than oxide dots with the same wetting layer thickness. Note that the solid symbols are the average value of measurement data. The upper and lower error bars are the maximum and minimum of the measured data.

not change with deposition time. The AFM images of oxide and oxynitride dots with similar wetting layer thicknesses are shown in Fig. 5a and c, respectively, and the corresponding cross-sectional morphologies are illustrated in Fig. 5b and d. Because the deposition rate for oxynitride is much higher than that for oxide, the deposition time for oxide was longer to have the same wetting layer thickness. From Fig. 5b and d, the oxynitride dots are more uniform in lateral size and height as compared to oxide dots. The dot densities of oxide and oxynitride dots are $2.1 \times 10^{9}$ and $1.7 \times 10^{9} \mathrm{~cm}^{-2}$, respectively. The higher dot density of oxide dots is owing to the overlapped small dots, as shown in Fig. 5b. The oxide and oxynitride dots have an average base width of 188 and $173 \mathrm{~nm}$, respectively (not including the overlapped small dots).

To have a metal-insulator-semiconductor (MIS) structure, aluminum was deposited on $1.5 \mathrm{~nm}$ LPD oxide and oxynitride films and was patterned to form MIS diodes ${ }^{10}$ with a circular area of 3 $\times 10^{-4} \mathrm{~cm}^{2}$. For MIS tunneling devices with insulators less than 3 $\mathrm{nm}$ thick, the minority carrier generation rate in the deep depletion region dominates the dark current at inversion bias. ${ }^{11}$ The LPDSiON device had a lower inversion current as compared to LPD-SiO ${ }_{2}$ device due to the $\mathrm{N}$ atoms at the interface to passivate the 

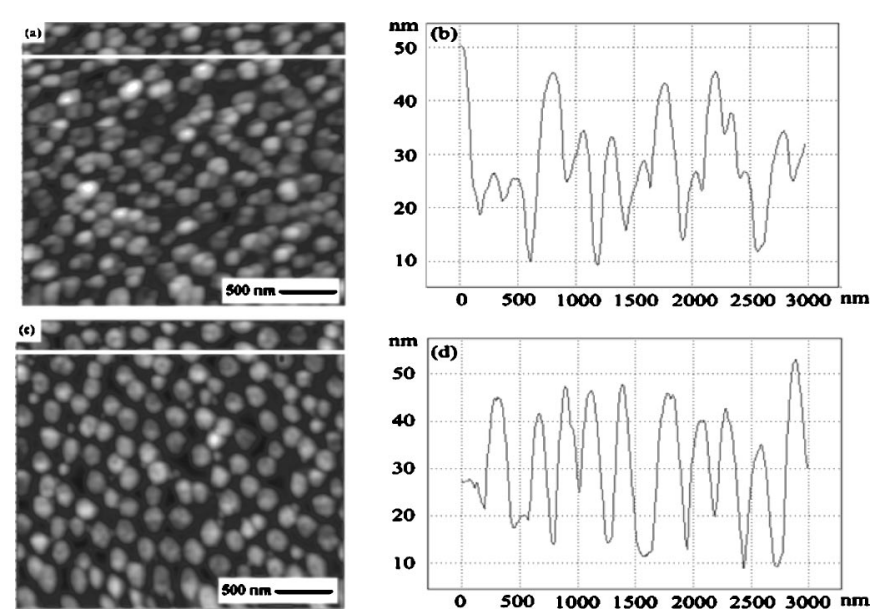

Figure 5. (a) AFM surface image of LPD oxide with $15 \mathrm{~nm}$ wetting layer thickness after 60 min growth. (b) Cross-sectional morphology of LPD oxide along the line in (a). (c) AFM surface image of LPD oxynitride (1 M $\mathrm{NH}_{4} \mathrm{OH}$ ) with $15 \mathrm{~nm}$ wetting layer thickness after 15 min growth. (d) Crosssectional morphology of LPD oxynitride along the line in (c).

interface states and to have a smaller interface state density $\left(D_{\mathrm{it}}\right)^{3}$.

In conclusion, the tensile strain on the $\mathrm{Si}$ cap above selfassembled quantum dots probably can enhance the etching rate of $\mathrm{Si}$ and have a preferential oxynitride deposition on Ge dots during
LPD. The recessed oxynitride dots are demonstrated with larger dot height as compared to oxide dots. Due to $\mathrm{N}$ atom passivation of the interface states, the device with oxynitride yields a lower dark current as compared to an oxide device.

\section{Acknowledgment}

This work is supported by National Science Council, Taiwan, R.O.C., under contract no. NSC 92-2120-E-002-006 and NSC 922215-E-002-007. TEM assistance by S. W. Lee, National Tsing Hua University, is also highly appreciated.

National Taiwan University assisted in meeting the publication costs of this article.

\section{References}

1. B.-C. Hsu, S. T. Chang, T.-C. Chen, P.-S. Kuo, P. S. Chen, and C. W. Liu, IEEE Electron Device Lett., 24, 318 (2003).

2. B.-C. Hsu, S. T. Chang, C.-R. Shie, C.-C. Lai, P. S. Chen, and C. W. Liu, Tech. Dig. - Int. Electron Devices Meet., 2002, 91.

3. B.-C. Hsu, W.-C. Hua, C.-R. Shie, K.-F. Chen, and C. W. Liu, Electrochem. SolidState Lett., 6, F9 (2003)

4. C. W. Liu, B.-C. Hsu, K.-F. Chen, M. H. Lee, C.-R. Shie, and P.-S. Chen, Appl. Phys. Lett., 82, 589 (2003)

5. D. J. Eaglesham and M. Cerullo, Phys. Rev. Lett., 64, 1943 (1990)

6. H. Nagayama, H. Honda, and H. Kawahara, J. Electrochem. Soc., 135, 2013 (1988).

7. J.-S. Chou and S.-C. Lee, Appl. Phys. Lett., 64, 1971 (1994).

8. M. K. Lee, S. Y. Lin, and J.-M. Shyr, J. Electrochem. Soc, 148, F1 (2001).

9. O. G. Schmidt and K. Eberl, Phys. Rev. B, 61, 13721 (2000).

10. C. W. Liu, W. T. Liu, M. H. Lee, W. S. Kuo, and B. C. Hsu, IEEE Electron Device Lett., 21, 307 (2000).

11. C.-H. Lin, B.-C. Hsu, M. H. Lee, and C. W. Liu, IEEE Trans. Electron Devices, 48 $2125(2001)$. 